respectively. The sensitivity and specificity of 3D USG with power Doppler were $84 \%$ and $88 \%$ respectively with area under the curve 0.96 ( $95 \% \mathrm{CI}=0.92-0.99)$. With simple rules, $11.9 \%$ $(10 / 84)$ cases were inconclusive of which 3D USG correctly discriminated 50\% malignant cases (3/6).

Conclusion: Both IOTA simple rules and 3D USG with power Doppler have a good ability to differentiate benign from malignant adnexal masses. 3D USG does not provide any added advantage over IOTA simple rules. However, the potential use of 3D USG as a second step test in inconclusive masses should be the subject of further evaluation.

Poster (004)

Gynecologic Pathology, Genetics and Epidemiology

https://doi.org/10.3802/jgo.2021.32.S1.004

\section{Copy number alterations feature of normal adjacent tissue as a novel biomarker for tumor burden in epithelial ovarian cancer patients}

Xiaopei, ${ }^{1}$ Lei Li, ${ }^{1,}$ ' Zhentian Kai, ${ }^{2}$ Lisha Huang, ${ }^{2}$ Ruijue Lin, ${ }^{3}$ Peng Luo, ${ }^{2}$ Hongsheng $\mathrm{He}^{2}{ }^{2}$ Lijun $\mathrm{Cai}^{2}$

'Peking Union Medical College Hospital, Beijing, China (lileigh@63.com) ${ }^{2}$ Shanghai Topgen Biomedical Technology Co., Ltd., Shanghai, China ${ }^{3}$ Zhejiang Topgen Clinical Laboratory Co, Ltd., Huzhou, China

Objective: Ovarian cancer typically presents high recurrence rate due to the propensity for ovarian cancer to disseminate throughout the peritoneum. Currently, there are limited studies based on genomic characteristics on histologically normal tissue adjacent to the tumor (NAT), we hereby characterized the genome-wide copy number alterations (CNAs) of NAT.

Methods: Seventeen blood samples, 10 tumor tissues (TT) and 10 NAT were collected from 10 patients with histologically confirmed diagnosis of ovarian cancer. All samples were sequenced to genome-wide coverage of $1 \times$. IchorCNA were used to determined tumor fraction (TF) in each samples with bin resolution of $1 \mathrm{Mb}$.

Results: The 3/10 (30\%) NAT samples revealed evidences of global CNA with TF of $10.72 \%, 4.77 \%$ and $25.67 \%$, respectively. $\mathrm{TF}$ in their corresponding ctDNA samples were $15.55 \%, 0 \%$, $13.08 \%$. The rest of patients have average TFs of $0.65 \%$ and $2.23 \%$ for ctDNA and NAT. A strong correlation between NAT and ctDNA in TF was observed. No significant difference in International Federation of Gynaecology and Obstetrics stages were observed between 2 subgroups. We subsequently analyzed CNA concordance among TT/NAT, TT/ctDNA, NAT/ctDNA pairs. NAT TF-positive patients showed high concordance (median values of $29.07 \%, 35.42 \%$ and $31.85 \%$ ) whereas NAT
TF-negative patients showed considerably lower concordance between their TT and NAT $(0 \%-5.42 \%)$.

Conclusion: Our results presented a subgroup of patients that exhibits high tumor fraction in both NAT and ctDNA. Their genomes altered in a much similar manner compared with low-TF patients. This phenomenon provided an early version of tumor aggressiveness and may becoming a promising biomarker for prognosis and recurrence.

Poster (005)

Epithelial Ovarian Cancer including Borderline Tumor https://doi.org/10.3802/jgo.2021.32.S1.005

\section{Correlation between preoperative $\mathrm{PCI}$ imaging and intraoperative $\mathrm{PCl}$ measurement in peritoneal carcinomatosis secondary to ovarian, tubal and primary peritoneal carcinoma}

\section{Arithat Patkhunpisit \\ Khon Kaen Hospital, Khon Kaen, Thailand (dpatkhun@gmail.com)}

Objective: To evaluate the correlation between preoperative and intraoperative percutaneous coronary intervention (PCI), the secondary objectives were to determine whether PCI could predict surgical oncologic outcomes and to identify factors statistically significant to the optimal surgical outcome.

Methods: In a prospective cohort study, women with advancedstage epithelial ovarian cancer or primary peritoneal cancer or fallopian tube cancer who underwent either primary cytoreductive surgery or interval debulking were included. The preoperative computed tomography (CT) scan findings and intraoperative measurement of the peritoneal carcinomatosis were evaluated by PCI and their correlation was determined using Spearman coefficient.

Results: A total of 55 women were enrolled, 52 patients were eligible and analyzed. Mean preoperative and intraoperative PCI were 5.04 and 7.27, orderly. Twenty-nine patients achieved optimal surgery $(55.8 \%)$. There is a moderate correlation between the PCI obtained from CT image and surgical findings $(r=0.510, p<0.001)$. The significant cutoff values of preoperative PCI and intraoperative PCI to predict optimal surgical outcome could be 7 and 8 respectively. In a univariate analysis, ECOG0 , preoperative CA125 $<416 \mathrm{U} / \mathrm{mL}$, preoperative $\mathrm{PCI}<7$ and intraoperative PCI $<8$ were significantly associated with the optimal surgical outcome. While with multivariate analysis, preoperative CA125 $<416 \mathrm{U} / \mathrm{mL}$ and intraoperative $\mathrm{PCI}<8$ were only independent factors related to the optimal surgery. Conclusion: Our correlation between preoperative PCI and 